MATHEMATICS OF COMPUTATION

Volume 68, Number 226, April 1999, Pages 749-765

S $0025-5718(99) 01029-7$

\title{
IRREGULAR SAMPLING, TOEPLITZ MATRICES, AND THE APPROXIMATION OF ENTIRE FUNCTIONS OF EXPONENTIAL TYPE
}

\author{
KARLHEINZ GRÖCHENIG
}

\begin{abstract}
In many applications one seeks to recover an entire function of exponential type from its non-uniformly spaced samples. Whereas the mathematical theory usually addresses the question of when such a function in $L^{2}(\mathbb{R})$ can be recovered, numerical methods operate with a finite-dimensional model. The numerical reconstruction or approximation of the original function amounts to the solution of a large linear system.

We show that the solutions of a particularly efficient discrete model in which the data are fit by trigonometric polynomials converge to the solution of the original infinite-dimensional reconstruction problem. This legitimatizes the numerical computations and explains why the algorithms employed produce reasonable results. The main mathematical result is a new type of approximation theorem for entire functions of exponential type from a finite number of values. From another point of view our approach provides a new method for proving sampling theorems.
\end{abstract}

A standard problem in many applications requires one to find a reconstruction of a function $f$ from a collection of samples $f\left(x_{n}\right)$. In most applications the assumption that $f$ is band-limited, or equivalently that $f$ is an entire function of exponential type, is well justified, and frequently the sampling points are non-uniformly spaced or distributed quite randomly. Then the mathematical problem is to find conditions under which $f$ can be reconstructed completely from its samples $f\left(x_{n}\right)$. This problem is almost completely understood thanks to the work of Duffin-Schaeffer, Beurling, Malliavin, Landau, Pavlov and others [5, 2, 3, 12, 13, 14, 15, 17]. Their work has provided deep insights into sets of uniqueness, Riesz bases, and sets of stable sampling. For reviews, more references, and different points of view of various aspects of the mathematical theory we refer to $[1,6,20]$.

In recent years the attention has focussed on practical solutions of the irregular sampling problem. See $[6,8]$ and the references cited there. Here the issues are the design of efficient and fast algorithms, explicit error estimates and stopping criteria.

Naturally, in any application the input for an algorithm must consist only of a finite number of data, in this case the samples $\left(x_{j}, f\left(x_{j}\right)\right), j=1, \ldots, N$, of a bandlimited function. Therefore numerically one deals with a finite-dimensional version of the infinite-dimensional problem.

Received by the editor October 25, 1996.

1991 Mathematics Subject Classification. Primary 30E05, 30E10, 42A10, 94A12.

Key words and phrases. Entire functions of exponential type, irregular sampling, Toeplitz matrices, approximation by trigonometric polynomials.

This work was partially supported by NSF grant DMS-9306430.

(C)1999 American Mathematical Society 
An algorithm for the sampling problem with input $\left\{\left(x_{j}, f\left(x_{j}\right)\right), j=1, \ldots, N\right\}$ can be interpreted as a sort of black box that produces some output $p(x)$ which is then claimed to be the "reconstruction" of $f$.

The goal of this paper is to clarify and analyze the relation between a natural finite-dimensional sampling model and the original infinite-dimensional sampling problem. In our experience the best numerical method consists of fitting the samples $f\left(x_{j}\right)$ in an interval $[-M, M]$ by a trigonometric polynomial $p_{M}$ of suitable degree and period $[7,9,16]$. Although in practice this method is very successful, it is not clear why $p_{M}$ should be a good approximation of $f$ on $[-M, M]$. Our main result demonstrates that under appropriate conditions the alleged reconstruction $p_{M}$ converges to the original function $f$, as the length $2 M$ of the interval increases. In this way we obtain a new theorem for the approximation of entire functions of exponential type from a finite number of samples.

The proof uses only real variable estimates and resembles the finite element or Ritz-Galerkin methods in differential equations. The first task is to understand the finite-dimensional problem of irregular sampling for trigonometric polynomials (Section 2) and to obtain estimates that are independent of the dimension. The heart of our approach is the uniform estimates on the condition number of certain Toeplitz matrices in Section 3. The other important feature is to understand how well entire functions can be approximated locally by trigonometric polynomials (Section 4). Section 5 contains the proof of the main theorem and its consequences. This method is of independent interest and could be useful in other contexts to obtain new sampling and approximation theorems, in particular in higher dimensions.

\section{RESUlts}

In order to formulate the results rigorously we need to describe the precise concepts and to remind the reader of the conventions adopted in this paper.

The Fourier transform of a function $f$ is normalized as

$$
\hat{f}(\xi)=\int_{\mathbb{R}} f(x) e^{-2 \pi i x \xi} d x,
$$

so that $f(x)=\int_{\mathbb{R}} \hat{f}(\xi) e^{2 \pi i x \xi} d \xi$. The $L^{2}$-norm is denoted by $\|f\|=\left(\int|f(x)|^{2} d x\right)^{\frac{1}{2}}$.

Our main object are entire functions of exponential type at most $\pi$ in $L^{2}(\mathbb{R})$, or equivalently the space of band-limited functions

$$
\mathcal{B}=\left\{f \in L^{2}(\mathbb{R}): \operatorname{supp} \hat{f} \subseteq\left[-\frac{1}{2}, \frac{1}{2}\right]\right\} .
$$

With this normalization the cardinal series takes the form

$$
f(x)=\sum_{n \in \mathbb{Z}} f(n) \frac{\sin \pi(x-n)}{\pi(x-n)} \quad \text { for all } f \in \mathcal{B},
$$

with convergence in $L^{2}(\mathbb{R})$ and uniformly on $\mathbb{R}[4]$, and Bernstein's inequality is

$$
\left\|f^{\prime}\right\| \leq \pi\|f\| \quad \text { for all } f \in \mathcal{B} \text {. }
$$

Furthermore, if $f \in \mathcal{B}$, then $f$ and all its derivatives vanish at infinity.

Any sampling sequence $\left\{x_{j}, j \in \mathbb{Z}\right\} \subseteq \mathbb{R}$ is ordered by magnitude, $x_{j}<x_{j+1}, j \in$ $\mathbb{Z}$, and $\lim _{j \rightarrow \pm \infty} x_{j}= \pm \infty$. In contrast to the classical treatments, we do not require a minimal distance between consecutive points.

Then the mathematical problem is to find $f \in \mathcal{B}$ from the given sampled values $\left\{f\left(x_{j}\right), j \in \mathbb{Z}\right\}$. However, in a realistic problem only samples in an interval $[-M, M]$, 
say, are given, and one wants to find a reasonable and computable approximation of $f(x)$ on $[-M, M]$ from the data set $\left\{f\left(x_{j}\right),\left|x_{j}\right| \leq M\right\}$. The analysis of the discrete irregular sampling problem [9] suggests an approximation by trigonometric polynomials that interpolate the data $f\left(x_{j}\right)$. Thus for any integer $M>0$ we look at the class of trigonometric polynomials

$$
\mathcal{P}_{M}=\left\{p: p(x)=\sum_{k=-M}^{M} a_{k} \frac{e^{2 \pi i k x /(2 M+1)}}{\sqrt{2 M+1}}, a_{k} \in \mathbb{C}\right\} .
$$

All polynomials in $\mathcal{P}_{M}$ have period $2 M+1$, and the normalization is taken so that the collection

$$
\phi_{M, k}(x)=\frac{1}{\sqrt{2 M+1}} e^{2 \pi i k x /(2 M+1)}, \quad k \in \mathbb{Z},
$$

is an orthonormal basis of $L^{2}\left(\left[-M-\frac{1}{2}, M+\frac{1}{2}\right]\right)$. In particular, we have

$$
\begin{gathered}
\|p\|_{2, M}:=\left(\int_{-M-\frac{1}{2}}^{M+\frac{1}{2}}|p(x)|^{2} d x\right)^{\frac{1}{2}}=\|a\|_{2}:=\left(\sum_{k \in \mathbb{Z}}\left|a_{k}\right|^{2}\right)^{\frac{1}{2}}, \\
\|p\|_{\infty} \leq\|p\|_{2, M} \quad \text { and } \quad\left\|p^{\prime}\right\|_{2, M} \leq \pi\|p\|_{2, M} .
\end{gathered}
$$

In the following we will write $I_{M}$ for the interval $\left[-M-\frac{1}{2}, M+\frac{1}{2}\right],\langle\cdot, \cdot\rangle_{M}$ and $\|\cdot\|_{2, M}$ for the inner product and the norm in $L^{2}\left(I_{M}\right)$. As in (7), finite sequences are always extended by zeros to sequences in $l^{2}(\mathbb{Z})$ with unambiguous norm $\|\cdot\|_{2}$.

Considering $p \in \mathcal{P}_{M}$ as a periodic tempered distribution on $\mathbb{R}$, its Fourier transform is $\hat{p}=\sum_{k=-M}^{M} a_{k} \delta_{k / M}$ and supp $\hat{p} \subseteq\left[-\frac{1}{2}, \frac{1}{2}\right]$. In view of the bandwidth of $f \in \mathcal{B}$ it is therefore natural to consider the trigonometric polynomials of period $2 M+1$ and order $M$ for the purpose of a local approximation of $f$.

To avoid boundary effects, we take all samples in $I_{M}$ and additionally the two adjacent samples. More formally, let

$$
K(M)=\max \left\{j: x_{j}<-M-\frac{1}{2}\right\} \text { and } L(M)=\min \left\{j: x_{j}>M+\frac{1}{2}\right\} ;
$$

then

$$
J_{M}=[K(M), L(M)] \cap \mathbb{Z} .
$$

Theorem 1. Suppose that the sampling set satisfies

$$
\sup _{j \in \mathbb{Z}}\left(x_{j+1}-x_{j}\right)=\delta<1
$$

and that $\left\{f\left(x_{j}\right), j \in \mathbb{Z}\right\}$ is given for some $f \in \mathcal{B}$. If $p_{M}$ denotes the unique trigonometric polynomial in $\mathcal{P}_{M}$ that solves the least squares problem

$$
\sum_{j \in J_{M}}\left|p_{M}\left(x_{j}\right)-f\left(x_{j}\right)\right|^{2} \frac{x_{j+1}-x_{j-1}}{2}=\text { minimum }
$$

where the minimum is taken over all $p \in \mathcal{P}_{M}$, then

$$
\lim _{M \rightarrow \infty} \int_{-M-\frac{1}{2}}^{M+\frac{1}{2}}\left|f^{(\ell)}(x)-p_{M}^{(\ell)}(x)\right|^{2} d x=0
$$

for all derivatives $\ell \geq 0$, and also $\lim _{M \rightarrow \infty} p_{M}(x)=f(x)$ uniformly on compact sets. 
In the next section we will see that the least squares problem (LSP) can be solved efficiently by the inversion of a Toeplitz matrix. At present the fastest and most accurate reconstruction algorithms for the irregular sampling problem of band-limited functions are based on the solution of (LSP) [7].

As a consequence of the proof we obtain the following sampling theorem for band-limited functions [8].

Corollary 1. If $\sup _{j \in \mathbb{Z}}\left(x_{j+1}-x_{j}\right)=\delta<1$, then for $f \in \mathcal{B}$

$$
(1-\delta)^{2}\|f\|^{2} \leq \sum_{j \in \mathbb{Z}}\left|f\left(x_{j}\right)\right|^{2} \frac{x_{j+1}-x_{j-1}}{2} \leq 4\|f\|^{2} .
$$

We refer to the review [6] for a discussion of this sampling theorem. While the assumption on the sampling set is somewhat restrictive, this is the only sampling theorem known to us with explicit constants. These constants provide an estimate for the condition number of the sampling problem, and determine the rate of convergence of iterative algorithms.

In the special case of regular sampling at the Nyquist rate the approximating polynomials can be written explicitly and computed easily. Then one obtains the following approximation theorem:

Corollary 2. Suppose that $\{f(j), j \in \mathbb{Z}\}$ are the samples of $f \in \mathcal{B}$. Define

$$
p_{M}(x)=\frac{1}{2 M+1} \sum_{j=-M}^{M} f(j) \frac{\sin \pi(x-j)}{\sin \frac{\pi(x-j)}{2 M+1}} .
$$

Then $\lim _{M \rightarrow \infty} \int_{|x| \leq M+\frac{1}{2}}\left|f^{(\ell)}(x)-p_{M}^{(\ell)}(x)\right|^{2} d x=0$ for all $\ell \geq 0$.

Diligent book-keeping of all estimates in the proof of Theorem 1 permits a more quantitative statement and shows that the approximation quality depends on the decay of $f$ at infinity.

Corollary 3. With the same assumptions as in Theorem 1 and $0<L \leq M$ we have

$$
\begin{aligned}
\int_{|x| \leq M+\frac{1}{2}}\left|f(x)-p_{M}(x)\right|^{2} d x & \\
\leq C(1-\delta)^{-2}\left\{\frac{L^{3}}{M^{2}}\|f\|^{2}+\|f\|\right. & \left(\int_{|x| \geq L}\left(|f(x)|^{2}+\left|f^{\prime}(x)\right|^{2}\right) d x\right)^{1 / 2} \\
& \left.+\sup _{\left|x-M-\frac{1}{2}\right| \leq 1}\left(|f( \pm x)|^{2}+\left|f^{\prime}( \pm x)\right|^{2}\right)\right\}
\end{aligned}
$$

where the constant $C$ does not depend on $f, \delta, L$, or $M$. In particular, if $f \in \mathcal{B}$ is rapidly decreasing, then $\left\|f-p_{M}\right\|_{2, M}^{2}=\mathcal{O}\left(M^{-2+\epsilon}\right)$ for all $\epsilon>0$.

\section{IRREGULAR SAMPLING OF TRIGONOMETRIC POLYNOMIALS}

To understand Theorem 1 we must first analyze the least squares problem (LSP) and find a solution method for any integer $M>0$. In view of our ultimate goal $\left\{x_{j}, j \in \mathbb{Z}\right\}$ is always a bi-infinite sequence, of which we consider only the samples in $J_{M} \subseteq[-M-1, M+1]$. 
We start with two simple observations.

1. If $p_{M}=\sum_{k=-M}^{M} a_{M}(k) \phi_{M, k} \in \mathcal{P}_{M}$ and $w_{j}>0$ is a sequence of weights, then

$$
\sum_{j \in J_{M}}\left|p_{M}\left(x_{j}\right)\right|^{2} w_{j}=\sum_{k=-M}^{M} \sum_{k=-M}^{M} a_{M}(k) \overline{a_{M}(l)} \sum_{j \in J_{M}} \frac{w_{j}}{2 M+1} e^{-2 \pi i(l-k) x_{j} /(2 M+1)} .
$$

Let $C_{M}$ denote the $(2 M+1) \times(2 M+1)$ positive semi-definite Toeplitz matrix with entries

$$
\left(C_{M}\right)_{k l}=\sum_{j \in J_{M}} \frac{w_{j}}{2 M+1} e^{-2 \pi i(k-l) x_{j} /(2 M+1)} \quad \text { for }|k|,|l| \leq M
$$

Then

$$
\sum_{j \in J_{M}}\left|p_{M}\left(x_{j}\right)\right|^{2} w_{j}=\left\langle C_{M} a_{M}, a_{M}\right\rangle .
$$

If the number of samples card $J_{M}$ exceeds $2 M+1$, then the evaluation map $p_{M} \rightarrow\left\{p\left(x_{j}\right), j \in J_{M}\right\}$ is one-to-one and the coefficient vector $a_{M} \in \mathbb{C}^{2 M+1}$ is uniquely determined. In this case $C_{M}$ is invertible.

2. Given $p_{M} \in \mathcal{P}_{M}$ with coefficients $a_{M}(k)$, the vector $C_{M} a_{M} \in \mathbb{C}^{2 M+1}$ contains only the samples $p_{M}\left(x_{j}\right)$, as is seen from the calculation

$$
\begin{gathered}
\left(C_{M} a_{M}\right)(k)=\sum_{l=-M}^{M} \sum_{j \in J_{M}} a_{M}(l) \frac{w_{j}}{2 M+1} e^{-2 \pi i(k-l) x_{j} /(2 M+1)} \\
=\sum_{j \in J_{M}} p_{M}\left(x_{j}\right) \frac{w_{j}}{\sqrt{2 M+1}} e^{-2 \pi i k x_{j} /(2 M+1)}
\end{gathered}
$$

These remarks lead to the following algorithm for the solution of (LSP).

Proposition 1 (An efficient algorithm). Let $\left\{\left(x_{j}, f\left(x_{j}\right)\right), j \in J_{M}\right\}$ be the given input, $w_{j}>0, j \in \mathbb{Z}$, a set of weights, and suppose card $J_{M} \geq 2 M+1$.

Step 1. Compute $b_{M} \in \mathbb{C}^{2 M+1}$, where

$$
b_{M}(k)=\sum_{j \in J_{M}} f\left(x_{j}\right) \frac{w_{j}}{\sqrt{2 M+1}} e^{-2 \pi i k x_{j} /(2 M+1)} \quad \text { for }|k| \leq M .
$$

Step 2. Compute $a_{M}=C_{M}^{-1} b_{M} \in \mathbb{C}^{2 M+1}$.

Step 3. Compute

$$
p_{M}(x)=\sum_{k=-M}^{M} a_{M}(k) \frac{e^{2 \pi i k x /(2 M+1)}}{\sqrt{2 M+1}} \in \mathcal{P}_{M} .
$$

Then for all $p \in \mathcal{P}_{M}, p \neq p_{M}$,

$$
\sum_{j \in J_{M}}\left|p_{M}\left(x_{j}\right)-f\left(x_{j}\right)\right|^{2} w_{j}<\sum_{j \in J_{M}}\left|p\left(x_{j}\right)-f\left(x_{j}\right)\right|^{2} w_{j} .
$$

Proof. We consider the subspace $\left\{\left(p\left(x_{j}\right)_{j \in J_{M}}: p \in \mathcal{P}_{M}\right\} \subseteq \mathbb{C}^{J_{M}}\right.$ with the inner product $\langle\mathbf{a}, \mathbf{b}\rangle_{w}=\sum_{j \in J_{M}} a_{j} \bar{b}_{j} w_{j}$ for $\mathbf{a}, \mathbf{b} \in \mathbb{C}^{J_{M}}$.

The solution of (LSP) is the orthogonal projection of the vector $\left\{f\left(x_{j}\right)\right.$, $\left.j \in J_{M}\right\} \in \mathbb{C}^{J_{M}}$ onto this subspace, and therefore it is sufficient to verify that

$$
\sum_{j \in J_{M}}\left(p_{M}\left(x_{j}\right)-f\left(x_{j}\right)\right) \overline{q_{M}\left(x_{j}\right)} w_{j}=0
$$


for every $q_{M} \in \mathcal{P}_{M}$ with coefficient vector $c_{M} \in \mathbb{C}^{2 M+1}$. But this is clear, since by (13) and Step 2

$$
\sum_{j \in J_{M}} p_{M}\left(x_{j}\right) \overline{q_{M}\left(x_{j}\right)} w_{j}=\left\langle C_{M} a_{M}, c_{M}\right\rangle=\left\langle b_{M}, c_{M}\right\rangle .
$$

On the other hand,

$$
\begin{array}{rl}
\sum_{j \in J_{M}} & f\left(x_{j}\right) \overline{q_{M}\left(x_{j}\right)} w_{j} \\
& =\sum_{k=-M}^{M} \overline{c_{M}(k)} \sum_{j \in J_{M}} f\left(x_{j}\right) \frac{w_{j}}{\sqrt{2 M+1}} e^{-2 \pi i k x_{j} /(2 M+1)}=\left\langle b_{M}, c_{M}\right\rangle .
\end{array}
$$

Remarks. 1. All steps of this algorithm can be implemented efficiently and computed quickly. Step 3 is just a discrete Fourier transform, and often Step 1 can also be computed with a fast Fourier transform. The computation of the entries of the Toeplitz matrix $C_{M}$ and its inversion can also be carried out by using a fast Fourier transform and preconditioning, and requires about $\mathcal{O}(2 M \log 2 M)$ operations. For a detailed discussion of this algorithm and a more organic derivation, as well as numerical simulations, we refer to [7]. Some spectacular applications are contained in Strohmer's thesis [18].

2. An alternative method to solve (LSP) has been proposed by Reichel, Ammar, and Gragg [16] and is based on unitary Hessenberg matrices and Szegö polynomials. Both methods seem to be equally efficient and require about $\mathcal{O}\left(2 M \cdot \operatorname{card} J_{M}\right)$ operations. For the theoretical discussion the transformation into a Toeplitz system is preferable, as will become clear in the following developments.

\section{A UNIFORM ESTIMATE OF THE CONDITION NUMBER}

The algorithm of Proposition 1 solves the least squares problem (LSP) for any choice of weights and for an arbitrary distribution of sampling points provided that $\left[-M-\frac{1}{2}, M+\frac{1}{2}\right]$ contains at least $2 M+1$ samples. However, if card $J_{M} \approx 2 M+1$ and if the sampling set contains large gaps, then the matrix of the problem is frequently very ill-conditioned [6]. This can be avoided by the appropriate choice of weights and by imposing the maximal gap condition (11).

Lemma 1. Let $\left\{x_{j}, j \in \mathbb{Z}\right\} \subseteq \mathbb{R}$ be an arbitrary sampling sequence and $\delta>0$. Define the weights $w_{j}>0$ by

$$
w_{j}=\min \left(\frac{1}{2}\left(x_{j}+x_{j+1}\right), x_{j}+\frac{\delta}{2}\right)-\max \left(\frac{1}{2}\left(x_{j}+x_{j-1}\right), x_{j}-\frac{\delta}{2}\right) .
$$

If $f$ is continuously differentiable and $K \leq L \in \mathbb{Z}$, then

$$
\sum_{j=K}^{L}\left|f\left(x_{j}\right)\right|^{2} w_{j} \leq 2 \int_{x_{K}-\frac{\delta}{2}}^{x_{L}+\frac{\delta}{2}}\left(|f(x)|^{2}+\frac{\delta^{2}}{\pi^{2}}\left|f^{\prime}(x)\right|^{2}\right) d x .
$$

Proof. Let $y_{j}=\max \left(\frac{1}{2}\left(x_{j}+x_{j-1}\right), x_{j}-\frac{\delta}{2}\right)$ and $z_{j}=\min \left(\frac{1}{2}\left(x_{j}+x_{j+1}\right), x_{j}+\frac{\delta}{2}\right)$, and let $\chi_{j}$ be the characteristic function of the interval $\left[y_{j}, z_{j}\right]$ containing $x_{j}$. Then 
$z_{j}-y_{j} \leq \delta$ and

$$
\left\|\sum_{j=K}^{L} f\left(x_{j}\right) \chi_{j}\right\|^{2}=\sum_{j=K}^{L}\left|f\left(x_{j}\right)\right|^{2} w_{j}
$$

We shall prove that

$$
\left\|\sum_{j=K}^{L}\left(f-f\left(x_{j}\right)\right) \chi_{j}\right\|^{2} \leq \frac{\delta^{2}}{\pi^{2}}\left\|\sum_{j=K}^{L} f^{\prime} \cdot \chi_{j}\right\|^{2}
$$

then (17) follows with the triangle inequality. Next we write

$$
\left\|\sum_{j=K}^{L}\left(f-f\left(x_{j}\right)\right) \chi_{j}\right\|^{2}=\sum_{j=K}^{L} \int_{y_{j}}^{z_{j}}\left|f(x)-f\left(x_{j}\right)\right|^{2} d x .
$$

To each term we apply Wirtinger's inequality [11] in the form

$$
\int_{a}^{b}|f(x)-f(c)|^{2} \leq \frac{4}{\pi^{2}} \max \left((b-c)^{2},(c-a)^{2}\right) \cdot \int_{a}^{b}\left|f^{\prime}(x)\right|^{2} d x .
$$

Since by definition $z_{j}-x_{j} \leq \delta / 2$ and $x_{j}-y_{j} \leq \delta / 2$, we obtain

$$
\sum_{j=K}^{L} \int_{y_{j}}^{z_{j}}\left|f(x)-f\left(x_{j}\right)\right|^{2} d x \leq \sum_{j=K}^{L} \frac{\delta^{2}}{\pi^{2}} \int_{y_{j}}^{z_{j}}\left|f^{\prime}(x)\right|^{2} d x \leq \frac{\delta^{2}}{\pi^{2}} \int_{y_{K}}^{z_{L}}\left|f^{\prime}(x)\right|^{2} d x,
$$

from which (19) and thus (17) follow.

Corollary 4 ([9]). Let $-M-\frac{1}{2} \leq x_{1}<\cdots<x_{r}<M+\frac{1}{2}$ and set $x_{0}=x_{r}-2 M-1$ and $x_{r+1}=x_{1}+2 M+1$. If $\max _{j=1, \ldots, r}\left(x_{j+1}-x_{j}\right)=\delta<1$, then for all $p \in \mathcal{P}_{M}$

$$
(1-\delta)^{2}\|p\|_{2, M}^{2} \leq \sum_{j=1}^{r}\left|p\left(x_{j}\right)\right|^{2} \frac{x_{j+1}-x_{j-1}}{2} \leq(1+\delta)^{2}\|p\|_{2, M}^{2}
$$

Proof. In this case $y_{j+1}=z_{j}$ and $\sum_{j=1}^{r} \chi_{j}=\chi_{\left[y_{1}, y_{1}+2 M+1\right]}$. The periodicity of $p$, (18), (20), and Bernstein's inequality imply

$$
\left(\int_{y_{1}}^{y_{1}+2 M+1}\left|p(x)-\sum_{j=1}^{r} p\left(x_{j}\right) \chi_{j}(x)\right|^{2} d x\right)^{1 / 2} \leq \frac{\delta}{\pi}\left\|p^{\prime}\right\|_{2, M} \leq \delta\|p\|_{2, M} .
$$

Now (21) follows with the triangle inequality.

Next we apply the corollary to segments of an infinite sampling sequence in order to derive estimates for the condition number of $C_{M}$. The following proposition is the key to understanding Theorem 1.

Proposition 2. Suppose $\sup _{j \in \mathbb{Z}}\left(x_{j+1}-x_{j}\right)=\delta<1$ and $C_{M}$ has the entries

$$
\left(C_{M}\right)_{k l}=\sum_{j \in J_{M}} \frac{x_{j+1}-x_{j-1}}{2(2 M+1)} e^{-2 \pi i(k-l) x_{j} /(2 M+1)} \quad \text { for }|k|,|l| \leq M .
$$

Then for all $a_{M} \in \mathbb{C}^{2 M+1}$ we have

$$
(1-\delta)^{2}\left\|a_{M}\right\|^{2} \leq\left\langle C_{M} a_{M}, a_{M}\right\rangle \leq 6\left\|a_{M}\right\|^{2} .
$$


In particular, the estimates

$$
\left\|C_{M}\right\|_{o p} \leq 6 \quad \text { and } \quad\left\|C_{M}^{-1}\right\|_{o p} \leq(1-\delta)^{-2}
$$

for the operator norm of $C_{M}$ are independent of the dimension $M$.

Proof. In view of (13) the assertion is equivalent to the inequalities

$$
(1-\delta)^{2}\|p\|_{2, M}^{2} \leq \sum_{j=1}^{r}\left|p\left(x_{j}\right)\right|^{2} \frac{x_{j+1}-x_{j-1}}{2} \leq 6\|p\|_{2, M}^{2}
$$

for all $p \in \mathcal{P}_{M}$. Since $p$ is $(2 M+1)$-periodic, we can consider the points $x_{K(M)}+$ $2 M+1 \in\left[-M-\frac{1}{2}, M+\frac{1}{2}\right]=I_{M}$ and $x_{L(M)}-2 M-1 \in I_{M}$ instead of $x_{K(M)}$ and $x_{L(M)}$, which were defined in (9). Rearranging the new set of points by magnitude and relabeling them, we obtain a new sequence $\left(\tilde{x}_{j}\right) \subseteq I_{M}$. This sequence coincides with the original segment $\left\{x_{j}, j \in J_{M}\right\}$ except for two points. The new sequence satisfies $\tilde{x}_{j+1}-\tilde{x}_{j} \leq \delta$ (it is exactly for this reason that the two points outside $I_{M}$ are included) and $\tilde{x}_{j+1}-\tilde{x}_{j-1} \leq x_{j+1}-x_{j-1} \leq \delta$, since we have added points to $I_{M}$. With the estimates of Corollary 4 we obtain

$$
\begin{aligned}
(1-\delta)^{2}\|p\|_{2, M}^{2} & \leq \sum_{j}\left|p\left(\tilde{x}_{j}\right)\right|^{2} \frac{\tilde{x}_{j+1}-\tilde{x}_{j-1}}{2} \\
& \leq \sum_{j \in J_{M}}\left|p\left(x_{j}\right)\right|^{2} \frac{x_{j+1}-x_{j-1}}{2} \\
& \leq \sum_{j}\left|p\left(\tilde{x}_{j}\right)\right|^{2} \frac{\tilde{x}_{j+1}-\tilde{x}_{j-1}}{2}+\delta^{2}\left|p\left(x_{K(M)}\right)\right|^{2}+\delta^{2}\left|p\left(x_{L(M)}\right)\right|^{2} \\
& \leq(1+\delta)^{2}\|p\|_{2, M}^{2}+2 \delta^{2}\|p\|_{\infty}^{2} .
\end{aligned}
$$

Since $\delta<1$ and $\|p\|_{\infty} \leq\|p\|_{2, M}$, the proposition is proved.

Lemma 2. Suppose that $\left\{x_{j}, j \in \mathbb{Z}\right\}$ is a sampling sequence with associated weights $w_{j}>0$, so that for some constant $\beta>0$

$$
\left\|C_{M}\right\|_{o p} \leq \beta \quad \text { uniformly for all } M>0 .
$$

Let $e_{M, j} \in \mathbb{C}^{2 M+1}$ for $j \in J_{M}$ be the vector defined by

$$
e_{M, j}(k)=\sqrt{\frac{w_{j}}{2 M+1}} e^{-2 \pi i k x_{j} /(2 M+1)} \quad \text { for }|k| \leq M .
$$

Then the following inequalities hold for all $g \in \mathbb{C}^{2 M+1}$ and all $\left(a_{j}\right)_{j \in J_{M}} \in \mathbb{C}^{J_{M}}$ :

$$
\sum_{j \in J_{M}}\left|\left\langle g, e_{M, j}\right\rangle\right|^{2} \leq \beta\|g\|_{2}^{2} \quad \text { and } \quad\left\|\sum_{j \in J_{M}} a_{j} e_{M, j}\right\|_{2}^{2} \leq \beta\|a\|_{2}^{2} .
$$

Proof. Here $\langle g, h\rangle=\sum_{k=-M}^{M} g(k) \overline{h(k)}$ is the standard inner product in $\mathbb{C}^{2 M+1}$. Therefore

$$
\begin{aligned}
& \sum_{j \in J_{M}}\left|\left\langle g, e_{M, j}\right\rangle\right|^{2}=\sum_{k=-M}^{M} \sum_{l=-M}^{M} g(l) \overline{g(k)} \sum_{j \in J_{M}} \overline{e_{M, j}(l)} e_{M, j}(k) \\
= & \sum_{k=-M}^{M} \sum_{l=-M}^{M} g(l) \overline{g(k)} \sum_{j \in J_{M}} \frac{w_{j}}{2 M+1} e^{-2 \pi i(k-l) x_{j} /(2 M+1)}=\left\langle C_{M} g, g\right\rangle
\end{aligned}
$$


and

$$
\left\langle C_{M} g, g\right\rangle \leq\left\|C_{M}\right\|_{o p}\|g\|_{2}^{2} \leq \beta\|g\|_{2}^{2} .
$$

The second inequality follows by duality:

$$
\begin{gathered}
\left\|\sum_{j \in J_{M}} a_{j} e_{M, j}\right\|_{2}^{2}=\sup _{\|g\|_{2}=1}\left|\sum_{j \in J_{M}} a_{j}\left\langle e_{M, j}, g\right\rangle\right|^{2} \\
\leq\left(\sum_{j \in J_{M}}\left|a_{j}\right|^{2}\right) \sup _{\|g\|_{2}=1} \sum_{j \in J_{M}}\left|\left\langle e_{M, j}, g\right\rangle\right|^{2} \leq \beta\|a\|_{2}^{2} \cdot \sup _{g}\|g\|_{2}^{2}=\beta\|a\|_{2}^{2} .
\end{gathered}
$$

\section{Estimates For PROJECTION OPERATORS}

Let $P$ be the orthogonal projection from $L^{2}(\mathbb{R})$ onto $\mathcal{B}$, and $P_{M}$ the orthogonal projection onto the $(2 M+1)$-dimensional space $\mathcal{P}_{M}$ spanned by the exponentials $\phi_{M, k}(x)=\frac{1}{\sqrt{2 M+1}} e^{2 \pi i k x /(2 M+1)}$ for $|k| \leq M$.

Since the least squares problem (LSP) approximates the original function $f$ by a trigonometric polynomial $p_{M} \in \mathcal{P}_{M}$ on $I_{M}$, we need some information on how and in what sense $P_{M}$ approximates $P$.

$P$ is given by the integral operator

$$
P f(x)=\int_{\mathbb{R}} f(y) \frac{\sin \pi(x-y)}{\pi(x-y)} d y .
$$

On the other hand, since $\phi_{M, k}$ is an orthonormal set in $L^{2}\left(I_{M}\right)$,

$$
\begin{gathered}
P_{M} f(x)=\sum_{k=-M}^{M}\left\langle f, \phi_{M, k}\right\rangle_{M} \phi_{M, k}(x) \\
=\frac{1}{2 M+1} \int_{I_{M}} f(y)\left(\sum_{k=-M}^{M} e^{2 \pi i k(x-y) /(2 M+1)}\right) d y \\
=\int_{-M-\frac{1}{2}}^{M+\frac{1}{2}} f(y) \frac{\sin \pi(x-y)}{(2 M+1) \sin \frac{\pi(x-y)}{2 M+1}} d y .
\end{gathered}
$$

Let

$$
K_{M}(x)=\sum_{k=-M}^{M}(2 M+1)^{-1 / 2} \phi_{M, k}(x)=\frac{\sin \pi x}{(2 M+1) \sin \frac{\pi x}{2 M+1}}
$$

be the convolution kernel of $P_{M}$. Then

$$
\left\|K_{M}\right\|_{2, M}^{2}=1 \quad \text { and } \quad\left|K_{M}(x)\right| \leq 1 \quad \text { for all } x .
$$

Since $K_{M}(x)$ converges uniformly to $\frac{\sin \pi x}{\pi x}$ and since $P$ and $P_{M}$ are orthogonal projections, a standard argument shows that $\lim _{M \rightarrow \infty} \chi_{I_{M}} \cdot P_{M} f=P f$ in $L^{2}(\mathbb{R})$ and uniformly on compact sets. However, since the kernels are given explicitly, we can do a little better and derive a quantitative estimate.

Lemma 3. For every $f \in \mathcal{B}$ and $0<L \leq M$ we have

$$
\left\|f-P_{M} f\right\|_{2, M}^{2} \leq c\|f\|\left(\frac{L^{3}}{M^{2}}\|f\|+\left(\int_{|y| \geq L}|f(y)|^{2} d y\right)^{\frac{1}{2}}\right)
$$


for a constant $c>0$. In particular, $\lim _{M \rightarrow \infty}\left\|f-P_{M} f\right\|_{2, M}^{2}=0$. Furthermore, $\lim _{M \rightarrow \infty} P_{M} f(x)=f(x)$ uniformly on compact sets.

Proof. Since $f=P f$ and since $P_{M}$ is an orthogonal projection on $L^{2}\left(I_{M}\right)$, we can write

$$
\left\|f-P_{M} f\right\|_{2, M}^{2}=\langle f, P f\rangle_{M}-\left\langle f, P_{M} f\right\rangle_{M}=\left\langle f,\left(P-P_{M}\right) f\right\rangle_{M} .
$$

Now fix $L, 0<L \leq M$, and $\chi_{L}=\chi_{[-L, L]}$, and insert the identity $f=f \chi_{L}+$ $f\left(1-\chi_{L}\right)$ into $(29)$. Then

$$
\begin{gathered}
\left\|f-P_{M} f\right\|_{2, M}^{2}=\left\langle f\left(1-\chi_{L}\right),\left(P-P_{M}\right) f\right\rangle_{M} \\
+\left\langle f \chi_{L},\left(P-P_{M}\right)\left(f-f \chi_{L}\right)\right\rangle_{M}+\left\langle f \chi_{L},\left(P-P_{M}\right)\left(f \chi_{L}\right)\right\rangle_{M}=I+I I+I I I .
\end{gathered}
$$

For the first term we obtain

$$
|I| \leq\left\|f\left(1-\chi_{L}\right)\right\|_{2, M}\left(\|P f\|_{2, M}+\left\|P_{M} f\right\|_{2, M}\right) \leq 2\|f\|\left(\int_{|y| \geq L}|f(y)|^{2} d y\right)^{1 / 2} .
$$

Similarly,

$$
|I I| \leq 2\|f\|\left(\int_{|y| \geq L}|f(y)|^{2} d y\right)^{1 / 2} .
$$

In the third term we estimate the difference of the integral kernels for small values of $x$ and $y$ :

$$
\begin{gathered}
\left|\frac{\sin \pi x}{\pi x}-K_{M}(x)\right|=\left|K_{M}(x)\left(\frac{\sin \frac{\pi x}{2 M+1}}{\frac{\pi x}{2 M+1}}-1\right)\right| \\
\leq\left|\frac{\sin \frac{\pi x}{2 M+1}}{\frac{\pi x}{2 M+1}}-1\right| \leq \frac{1}{3}\left(\frac{\pi x}{2 M+1}\right)^{2}
\end{gathered}
$$

since $\left\|K_{M}\right\|_{\infty} \leq 1$. Therefore

$$
\begin{aligned}
&|I I I|=\left|\int_{|x| \leq L} \int_{|y| \leq L} f(x) \overline{f(y)}\left(\frac{\sin \pi(x-y)}{\pi(x-y)}-K_{M}(x-y)\right) d x d y\right| \\
& \leq \frac{\pi^{2}}{3(2 M+1)^{2}} \int_{|x| \leq L} \int_{|y| \leq L}|f(x) f(y)|(x-y)^{2} d x d y \\
& \leq \frac{\pi^{2}}{3(2 M+1)^{2}}\|f\|^{2}\left(\int_{|x| \leq L} \int_{|y| \leq L}(x-y)^{4} d x d y\right)^{\frac{1}{2}} \leq \frac{\pi^{2}}{3(2 M+1)^{2}}(2 L)^{3}\|f\|^{2} .
\end{aligned}
$$

Combining these estimates yields, for any $0<L \leq M$,

$$
\left\|f-P_{M} f\right\|_{2, M}^{2} \leq c\|f\|\left(\frac{L^{3}}{M^{2}}\|f\|+\left(\int_{|y| \geq L}|f(y)|^{2} d y\right)^{\frac{1}{2}}\right)
$$

for the constant $c=\max \left(2 \pi^{2} / 3,4\right)$. Choosing $L=M^{\alpha}, 0<\alpha<2 / 3$, yields $\lim _{M \rightarrow \infty}\left\|f-p_{M}\right\|_{2, M}^{2}=0$.

The argument for uniform convergence on compact sets is similar and is left to the reader.

We need one more property of the projections $P_{M}$. 
Lemma 4. (a) For any differentiable function $f$ we have

$$
\left(P_{M} f\right)^{\prime}(x)=P_{M} f^{\prime}(x)-\left(f\left(M+\frac{1}{2}\right)-f\left(-M-\frac{1}{2}\right)\right) K_{M}\left(x+M+\frac{1}{2}\right) .
$$

(b) If $0<L \leq M / 2$ and $f \in L^{2}(\mathbb{R})$, then

$$
\sup _{\left|x-M-\frac{1}{2}\right| \leq 1}\left|P_{M} f( \pm x)\right|^{2} \leq \frac{L}{M^{2}}\|f\|^{2}+\int_{|y| \geq L}|f(y)|^{2} d y .
$$

Proof. (a) follows from the computation

$$
\begin{gathered}
\left(P_{M} f\right)^{\prime}(x)=\int_{-M-\frac{1}{2}}^{M+\frac{1}{2}} f(y) \frac{d}{d x} K_{M}(x-y) d y=-\int_{-M-\frac{1}{2}}^{M+\frac{1}{2}} f(y) \frac{d}{d y} K_{M}(x-y) d y \\
=\int_{-M-\frac{1}{2}}^{M+\frac{1}{2}} f^{\prime}(y) K_{M}(x-y) d y-\left.f(y) K_{M}(x-y)\right|_{y=-M-\frac{1}{2}} ^{M+\frac{1}{2}} \\
=P_{M} f^{\prime}(x)-\left(f\left(M+\frac{1}{2}\right) K_{M}\left(x-M-\frac{1}{2}\right)-f\left(-M-\frac{1}{2}\right) K_{M}\left(x+M+\frac{1}{2}\right)\right) .
\end{gathered}
$$

For (b) we write

$$
\begin{aligned}
& \left|P_{M} f( \pm x)\right|^{2}=\left|\int_{-M-\frac{1}{2}}^{M+\frac{1}{2}} f(y) K_{M}( \pm x-y) d y\right|^{2} \\
& \quad \leq \int_{|y| \leq L}|f(y)|^{2} d y \cdot \int_{|y| \leq L}\left|K_{M}( \pm x-y)\right|^{2} d y \\
& +\int_{|y| \geq L}|f(y)|^{2} d y \cdot \int_{L \leq|y| \leq M+\frac{1}{2}}\left|K_{M}( \pm x-y)\right|^{2} d y .
\end{aligned}
$$

We take into account that for $x=M+\frac{1}{2}+\xi$ and $|\xi| \leq 1$

$$
\left|K_{M}( \pm x-y)\right|^{2}=\left|K_{M}\left(M+\frac{1}{2} \pm \xi-y\right)\right|^{2}=\left|\frac{\cos \pi( \pm \xi-y)}{(2 M+1) \cos \frac{\pi( \pm \xi-y)}{2 M+1}}\right|^{2} \leq \frac{2}{(2 M+1)^{2}}
$$

provided that $\frac{\pi| \pm \xi-y|}{2 M+1} \leq \frac{\pi(L+1)}{2 M+1} \leq \pi / 4$. Altogether we obtain

$$
\sup _{\left|x-M-\frac{1}{2}\right| \leq 1}\left|P_{M} f( \pm x)\right|^{2} \leq \frac{4 L}{(2 M+1)^{2}}\|f\|^{2}+\int_{|y| \geq L}|f(y)|^{2} d y .
$$

\section{Proof OF THE MAIN THEOREM}

We are now in a position to prove Theorem 1 and its consequences.

Recall that from the samples $f\left(x_{j}\right)$ of $f \in \mathcal{B}$ we construct a trigonometric polynomial $p_{M}=\sum_{k=-M}^{M} a_{M}(k) \phi_{M, k} \in \mathcal{P}_{M}$ that approximates the samples in $[-M, M]$ optimally. The solution of the associated least square problem required the inversion of the system's Toeplitz matrix $C_{M}$.

The proof that $p_{M}$ converges to $f$ does not depend on the particular assumptions on the sampling set. All that is required are the uniform bounds on the condition numbers of $C_{M}$ established in Proposition 2. We state this as a separate theorem, since this type of conclusion could be useful in other situations. 
Theorem 2. Suppose that $\left\{x_{j}, j \in \mathbb{Z}\right\}$ is a sampling sequence with associated weights chosen as in (16), $f \in \mathcal{B}$ and that for any $M \in \mathbb{Z}, M>0, p_{M} \in \mathcal{P}_{M}$ is the solution of the least squares problem (LSP). If there exist constants $0<\alpha \leq \beta$, so that the spectrum of $C_{M}$ satisfies

$$
\sigma\left(C_{M}\right) \subseteq[\alpha, \beta] \quad \text { uniformly for all } M>0,
$$

then $\lim _{M \rightarrow \infty} p_{M}(x)=f(x)$ converges uniformly on compact sets and

$$
\lim _{M \rightarrow \infty} \int_{-M-\frac{1}{2}}^{M+\frac{1}{2}}\left|f^{(\ell)}(x)-p_{M}^{(\ell)}(x)\right|^{2} d x=0
$$

for all $\ell \geq 0$. Furthermore,

$$
\alpha\|f\|^{2} \leq \sum_{j \in \mathbb{Z}}\left|f\left(x_{j}\right)\right|^{2} w_{j} \leq 4\|f\|^{2} .
$$

According to Proposition 2, the hypothesis (11) on the maximal gap implies $\sigma\left(C_{M}\right) \subseteq\left[(1-\delta)^{2}, 6\right]$. Therefore, Theorem 1 and Corollary 1 are immediate consequences of Theorem 2 .

Proof. Step 1. In order to compute

$$
\int_{-M-\frac{1}{2}}^{M+\frac{1}{2}}\left|f(x)-p_{M}(x)\right|^{2} d x
$$

we expand $f_{M}:=f \cdot \chi_{I_{M}}$ into a Fourier series

$$
f_{M}=\sum_{k \in \mathbb{Z}} \hat{f}_{M}(k) \phi_{M, k} \quad \text { for }|x| \leq M+\frac{1}{2},
$$

where $\hat{f}_{M}(k)=\left\langle f, \phi_{M, k}\right\rangle_{M}$. Then $f-P_{M} f=\sum_{|k|>M} \hat{f}_{M}(k) \phi_{M, k}$ is orthogonal to $\mathcal{P}_{M}$ in $L^{2}\left(I_{M}\right)$, and we can write

$$
\left\|f-p_{M}\right\|_{2, M}^{2}=\left\|f-P_{M} f\right\|_{2, M}^{2}+\left\|P_{M} f-p_{M}\right\|_{2, M}^{2}=R_{M}+A_{M}
$$

The remainder $R_{M}$ tends to zero by Lemma 3 .

Step 2. The estimate for $A_{M}$ is harder and makes fundamental use of the lower bound on $C_{M}$. By slight abuse of notation we still denote the vector $\left\{\hat{f}_{M}(k),|k| \leq\right.$ $M\}$ by $\hat{f}_{M} \in \mathbb{C}^{2 M+1}$. Since by construction (Proposition 1) $\hat{p}_{M}(k)=a_{M}(k)=$ $\left(C_{M}^{-1} b_{M}\right)(k)$ for $|k| \leq M$, and $\hat{p}_{M}(k)=0$ otherwise, we obtain

$$
\begin{gathered}
A_{M}=\left\|P_{M} f-p_{M}\right\|_{2, M}^{2}=\left\|\hat{f}_{M}-a_{M}\right\|_{2}^{2}=\left\|C_{M}^{-1}\left(C_{M} \hat{f}_{M}-b_{M}\right)\right\|_{2}^{2} \\
\leq \alpha^{-2}\left\|C_{M} \hat{f}_{M}-b_{M}\right\|_{2}^{2}
\end{gathered}
$$


Because $C_{M}$ and $b_{M}$ are known explicitly, we can rewrite this expression and manipulate it so that Lemma 2 is applicable:

$$
\begin{aligned}
& \left\|C_{M} \hat{f}_{M}-b_{M}\right\|_{2}^{2}=\sum_{k=-M}^{M}\left|\sum_{l=-M}^{M}\left(C_{M}\right)_{k l} \hat{f}_{M}(l)-b_{M}(k)\right|^{2} \\
& =\sum_{k=-M}^{M} \mid \sum_{l=-M}^{M} \sum_{j \in J_{M}} \frac{w_{j}}{2 M+1} e^{-2 \pi i(k-l) x_{j} /(2 M+1)} \hat{f}_{M}(l) \\
& \quad-\left.\sum_{j \in J_{M}} \frac{w_{j}}{\sqrt{2 M+1}} f\left(x_{j}\right) e^{-2 \pi i k x_{j} /(2 M+1)}\right|^{2} \\
& =\sum_{k=-M}^{M} \mid \sum_{j \in J_{M}} \sqrt{w_{j}}\left\{f\left(x_{j}\right)-\frac{1}{\sqrt{2 M+1}} \sum_{l=-M}^{M} e^{2 \pi i l x_{j} /(2 M+1)} \hat{f}_{M}(l)\right\} \\
& \leq\left.\beta \sum_{j \in J_{M}} w_{j}\left|f\left(x_{j}\right)-\frac{1}{\sqrt{2 M+1}} \sum_{l=-M}^{M} e^{2 \pi i l x_{j} /(2 M+1)} \hat{f}_{M}(l)\right|^{-2 \pi i k x_{j} /(2 M+1)}\right|^{2}
\end{aligned}
$$

where the last inequality follows from Lemma 2. Finally, the identification

$$
\frac{1}{\sqrt{2 M+1}} \sum_{l=-M}^{M} e^{2 \pi i l x_{j} /(2 M+1)} \hat{f}_{M}(l)=\sum_{l=-M}^{M}\left\langle f, \phi_{M, l}\right\rangle_{M} \phi_{M, l}\left(x_{j}\right)=P_{M} f\left(x_{j}\right)
$$

leads to the estimate

$$
A_{M} \leq \frac{\beta}{\alpha^{2}} \sum_{j \in J_{M}} w_{j}\left|f\left(x_{j}\right)-P_{M} f\left(x_{j}\right)\right|^{2}
$$

Step 3. We apply Lemma 1 with $\delta=1$ to $h_{M}=f-P_{M} f$ and obtain

$$
\begin{aligned}
\sum_{j \in J_{M}} w_{j}\left|h_{M}\left(x_{j}\right)\right|^{2} & \leq 2 \int_{-M-\frac{3}{2}}^{M+\frac{3}{2}}\left(\left|h_{M}(x)\right|^{2}+\frac{1}{\pi^{2}}\left|h_{M}^{\prime}(x)\right|^{2}\right) d x \\
& =\int_{I_{M}}+\int_{M+\frac{1}{2} \leq|x| \leq M+\frac{3}{2}} .
\end{aligned}
$$

Here $\int_{I_{M}}\left|f-P_{M} f\right|^{2}=o(1)$ by Lemma 3. For $\int_{I_{M}}\left|h_{M}^{\prime}\right|^{2}$ we use Lemma 4 and obtain

$$
\left\|\left(f-P_{M} f\right)^{\prime}\right\|_{2, M}^{2} \leq 2\left\|f^{\prime}-P_{M} f^{\prime}\right\|_{2, M}^{2}+2\left|f\left(-M-\frac{1}{2}\right)-f\left(M+\frac{1}{2}\right)\right|^{2}\left\|K_{M}\right\|_{2, M}^{2},
$$

which also converges to zero by Lemma 3 .

Step 4 . The rest is estimated coarsely by taking suprema:

$$
\begin{gathered}
\int_{M+\frac{1}{2} \leq|x| \leq M+\frac{3}{2}}\left(\left|h_{M}(x)\right|^{2}+\frac{1}{\pi^{2}}\left|h_{M}^{\prime}(x)\right|^{2}\right) d x \\
\leq 2 \sup _{M+\frac{1}{2} \leq|x| \leq M+\frac{3}{2}}\left(|f(x)|^{2}+\left|f^{\prime}(x)\right|^{2}+\left|P_{M} f(x)\right|^{2}+\left|\left(P_{M} f\right)^{\prime}(x)\right|^{2}\right) .
\end{gathered}
$$


Since $f \in \mathcal{B}$, all terms under the supremum tend to zero, where we have to use Lemma 4 to handle $P_{M} f$ and $\left(P_{M} f\right)^{\prime}$.

Combining these estimates, we have proved so far that $\int_{-M-\frac{1}{2}}^{M+\frac{1}{2}}\left|f(x)-p_{M}(x)\right|^{2}$ tends to zero, as $M \rightarrow \infty$.

Step 5. Pointwise convergence. We return to the beginning of the proof and write

$$
\begin{gathered}
\left|f(x)-p_{M}(x)\right| \leq\left|f(x)-P_{M} f(x)\right|+\left|P_{M} f(x)-p_{M}(x)\right| \\
=\left|f(x)-P_{M} f(x)\right|+\left|\frac{1}{\sqrt{2 M+1}} \sum_{k=-M}^{M}\left(\hat{f}_{M}(k)-a_{M}(k)\right) e^{2 \pi i k x /(2 M+1)}\right| \\
\leq\left|f(x)-P_{M} f(x)\right|+\left(\sum_{k=-M}^{M}\left|\hat{f}_{M}(k)-a_{M}(k)\right|^{2}\right)^{1 / 2} .
\end{gathered}
$$

The first term converges to zero uniformly on compact sets by Lemma 3, whereas the second term does not depend on $x$ and converges to zero by steps $1-4$.

Step 6. Convergence of the derivatives. Again we write for $\ell \geq 1$, as in (34),

$$
\left\|f^{(\ell)}-p_{M}^{(\ell)}\right\|_{2, M}^{2}=\left\|f^{(\ell)}-P_{M} f^{(\ell)}\right\|_{2, M}^{2}+\left\|P_{M} f^{(\ell)}-p_{M}^{(\ell)}\right\|_{2, M}^{2} .
$$

Since $f^{(\ell)} \in \mathcal{B}$, the first term converges to zero by Lemma 3. Iterating the formula in Lemma 4 (a), we obtain

$$
\left(P_{M} f\right)^{(\ell)}(x)=P_{M} f^{(\ell)}(x)+\sum_{k=0}^{\ell-1}\left(f^{(k)}\left(M+\frac{1}{2}\right)-f^{(k)}\left(-M-\frac{1}{2}\right)\right) K_{M}\left(M+\frac{1}{2}+x\right) .
$$

Therefore, using (28) and Bernstein's inequality

$$
\begin{gathered}
\left\|P_{M} f^{(\ell)}-p_{M}^{(\ell)}\right\|_{2, M}^{2} \leq 2\left(\left\|\left(P_{M} f\right)^{(\ell)}-p_{M}^{(\ell)}\right\|_{2, M}^{2}+\left|\sum_{k=0}^{\ell-1}\left(f^{(k)}\left(M+\frac{1}{2}\right)-f^{(k)}\left(-M-\frac{1}{2}\right)\right)\right|^{2}\right) \\
\leq 2\left(\pi^{2 \ell}\left\|P_{M} f-p_{M}\right\|_{2, M}^{2}+\left|\sum_{k=0}^{\ell-1}\left(f^{(k)}\left(M+\frac{1}{2}\right)-f^{(k)}\left(-M-\frac{1}{2}\right)\right)\right|^{2}\right) .
\end{gathered}
$$

Using Lemma 3 and $f \in \mathcal{B}$ again, the convergence of the derivatives is verified.

Step 7. Norm equivalence. The upper bound in (33) is a consequence of the particular choice of the weights $w_{j}$ and follows easily from Lemma 1 with $\delta=1$ :

$$
\begin{gathered}
\sum_{j \in J_{M}}\left|f\left(x_{j}\right)\right|^{2} w_{j} \leq 2 \int_{-M-\frac{3}{2}}^{M+\frac{3}{2}}\left(|f(x)|^{2}+\frac{1}{\pi^{2}}\left|f^{\prime}(x)\right|^{2}\right) d x \\
\leq 2\left(\|f\|^{2}+\frac{1}{\pi^{2}}\left\|f^{\prime}\right\|^{2}\right) \leq 4\|f\|^{2} \quad \forall M>0 .
\end{gathered}
$$

For the lower bound we show first that

$$
\lim _{M \rightarrow \infty} \sum_{j \in J_{M}}\left|f\left(x_{j}\right)-p_{M}\left(x_{j}\right)\right|^{2} w_{j}=0 .
$$


As in Step 3 we estimate

$$
\begin{aligned}
\sum_{j \in J_{M}} \mid & f\left(x_{j}\right)-\left.p_{M}\left(x_{j}\right)\right|^{2} w_{j} \leq \int_{-M-\frac{3}{2}}^{M+\frac{3}{2}}\left(\left|f-p_{M}\right|^{2}+\left|f^{\prime}-p_{M}^{\prime}\right|^{2}\right) \\
\leq & \left\|f-p_{M}\right\|_{2, M}^{2}+\left\|f^{\prime}-p_{M}^{\prime}\right\|_{2, M}^{2} \\
& +\sup _{\left|x-M-\frac{1}{2}\right| \leq 1}\left(|f( \pm x)|^{2}+\left|f^{\prime}( \pm x)\right|^{2}+\left|p_{M}( \pm x)\right|^{2}+\left|p_{M}^{\prime}( \pm x)\right|^{2}\right) .
\end{aligned}
$$

In view of the preceding steps we only have to treat the suprema over $p_{M}$ and $p_{M}^{\prime}$. Because of

$$
\left|p_{M}(x)\right| \leq\left|p_{M}(x)-P_{M} f(x)\right|+\left|P_{M} f(x)\right| \leq\left\|p_{M}-P_{M} f\right\|_{2, M}+\left|P_{M} f(x)\right| .
$$

Lemma 4 and Steps $1-4$ yield

$$
\lim _{M \rightarrow \infty} \sup _{\left|x-M-\frac{1}{2}\right| \leq 1}\left|p_{M}(x)\right|^{2}=0 .
$$

The supremum of $p_{M}^{\prime}$ is treated in a similar fashion.

Now, given any $\epsilon>0$, choose $M$ large enough so that $\left\|f-p_{M}\right\|_{2, M}<\epsilon \alpha^{-\frac{1}{2}}\|f\|$, $\sum_{j \in J_{M}}\left|f\left(x_{j}\right)-p_{M}\left(x_{j}\right)\right|^{2} w_{j}<\epsilon^{2}\|f\|^{2}$ and $\|f\|-\|f\|_{2, M}<\epsilon \alpha^{-\frac{1}{2}}\|f\|$. Then

$$
\begin{gathered}
\left(\sum_{j \in \mathbb{Z}}\left|f\left(x_{j}\right)\right|^{2} w_{j}\right)^{1 / 2} \geq\left(\sum_{j \in J_{M}}\left|f\left(x_{j}\right)\right|^{2} w_{j}\right)^{1 / 2} \\
\geq\left(\sum_{j \in J_{M}}\left|p_{M}\left(x_{j}\right)\right|^{2} w_{j}\right)^{1 / 2}-\left(\sum_{j \in J_{M}}\left|f\left(x_{j}\right)-p_{M}\left(x_{j}\right)\right|^{2} w_{j}\right)^{1 / 2} \\
\geq \sqrt{\alpha}\left\|p_{M}\right\|_{2, M}-\epsilon\|f\| \geq \sqrt{\alpha}\left(\|f\|_{2, M}-\left\|p_{M}-f\right\|_{2, M}\right)-\epsilon\|f\| \\
\geq \sqrt{\alpha}\left(\|f\|-\epsilon \alpha^{-\frac{1}{2}}\|f\|-\epsilon \alpha^{-\frac{1}{2}}\|f\|\right)-\epsilon\|f\|=(\sqrt{\alpha}-3 \epsilon)\|f\| .
\end{gathered}
$$

This completes the proof of Theorem 2 .

Proof of Corollary 2. The given polynomial actually interpolates the given data $\{f(j),|j| \leq M\}$. In this case the system matrix $C_{M}$ is the $(2 M+1) \times(2 M+1)$ identity matrix, as is seen from

$$
\left(C_{M}\right)_{k l}=\sum_{j=-M}^{M} \frac{1}{2 M+1} e^{-2 \pi i(k-l) j /(2 M+1)}=\delta_{k l} .
$$

Thus Theorem 2 is applicable.

Proof of Corollary 3. The main contributions to the error $\left\|f-p_{M}\right\|_{2, M}^{2}$ are the terms

$$
\begin{gathered}
\left\|f-P_{M} f\right\|_{2, M}^{2}, \\
\sup _{\left|x-M-\frac{1}{2}\right| \leq 1}\left(|f( \pm x)|^{2}+\left|f^{\prime}( \pm x)\right|^{2}\right),
\end{gathered}
$$

and

$$
\sup _{\left|x-M-\frac{1}{2}\right| \leq 1}\left(\left|P_{M} f( \pm x)\right|^{2}+\left|\left(P_{M} f\right)^{\prime}( \pm x)\right|^{2}\right.
$$


as follows from (34), (36) and (37). The estimate of the corollary follows from Lemmas 3 and 4 . The maximal gap condition yields the condition number of order $(1-\delta)^{-2}$ (Proposition 2).

\section{Conclusion}

Theorem 2 provides a natural strategy to prove theorems about the approximation and reconstruction of entire functions of exponential type. It reduces these questions to a finite-dimensional problem about the spectrum of certain Toeplitz matrices and the sampling of trigonometric polynomials. The method outlined in Theorem 2 could provide a new proof of the theorems of Duffin-Schaeffer [5] and Beurling-Landau [2,13], with the additional benefit of an explicit and efficient numerical algorithm attached to the proof.

Let $d(X)=\lim _{M \rightarrow \infty} \frac{1}{2 M} \min _{r>0} \sum_{j \in \mathbb{Z}} \chi_{[r-M, r+M]}\left(x_{j}\right)$ denote the Beurling density of a sampling set $X=\left\{x_{j}, j \in \mathbb{Z}\right\}$. Then Beurling's theorem states that if $d(X)>1$, then an inequality of the form (33) holds. Conversely, if (33) holds for some sampling set $X$, then $d(X) \geq 1$.

The condition $d(X)>1$ implies that for large $M$ every interval of length $2 M$ contains at least $[2 M d(X)] \geq 2 M+1$ samples. Thus, as in Proposition 1 the system matrix $C_{M}$ is invertible, the least squares problem (LSP) has a unique solution. In view of Theorem 2 it is therefore plausible to conjecture that the hypothesis $d(X)>1$ implies uniform estimates on the condition number of $C_{M}$. We hope to return to this question.

Theorem 2 and its proof carry over to higher dimensions with only minor modifications in the notation [10]. Theorem 2 allows us to reduce the sampling problem for multivariate band-limited functions to a finite-dimensional problem about trigonometric polynomials, and thus offers a new strategy for establishing irregular sampling theorems in higher dimensions as well as corresponding numerical algorithms.

\section{REFERENCES}

[1] J. J. Benedetto. Frame decompositions, sampling, and uncertainty principle inequalities. In "Wavelets: Mathematics and Applications", J. Benedetto, M. Frazier, eds., pp. 247-304, CRC Press, 1993. MR 94i:94005

[2] A. Beurling. Collected Works. Vol. 2, Harmonic Analysis, L. Carleson, ed., Birkhäuser, Boston, 1989, pp. 341-365. MR 92k:01046b

[3] A. Beurling, P. Malliavin. On the closure of characters and the zeros of entire functions. Acta Math. 118 (1967), 79-95. MR 35:654

[4] P. L. Butzer, W. Splettstößer, R. Stens. The sampling theorem and linear prediction in signal analysis. Jber.d. Dt. Math.-Verein. 90 (1987), 1-70. MR 89b:94006

[5] R. Duffin, A. Schaeffer. A class of nonharmonic Fourier series. Trans. Amer. Math. Soc. 72(1952), 341-366. MR 13:839a

[6] H. G. Feichtinger, K. Gröchenig. Theory and practice of irregular sampling. In "Wavelets: Mathematics and Applications", J. Benedetto, M. Frazier, eds., pp. 305-363, CRC Press, 1993. MR 94i:94008

[7] H. G. Feichtinger, K. Gröchenig, T. Strohmer. Efficient numerical methods in non-uniform sampling theory. Num. Math. 69(4) (1995), 423-440. MR 95j:65011

[8] K. Gröchenig, Reconstruction algorithms in irregular sampling. Math. Comp. 59 (1992), 181194. MR 93a: 41025

[9] K. Gröchenig, A discrete theory of irregular sampling. Lin. Alg. Appl. 193 (1993), 129-150. MR 94m:94005

[10] K. Gröchenig. Finite and Infinite-Dimensional Models of Non-Uniform Sampling. Proc. SampTA 97, Aveiro, Portugal, June 1997, pp. 285-290. 
[11] G. Hardy, J. E. Littlewood, G. Pólya. Inequalities. 2nd Ed., Cambridge Univ. Press. 1952. MR 13:727e

[12] S. Jaffard. A density criterion for frames of complex exponentials. Michigan Math. J. 38 (1991), 339-348. MR 92i:42001

[13] H. Landau. Necessary density conditions for sampling and interpolation of certain entire functions. Acta Math. 117 (1967), 37-52. MR 36:5604

[14] H. Landau. Extrapolating a band-limited function from its samples taken in a finite interval. IEEE Trans. Information Theory 32(4) (1986), 464-470.

[15] B. S. Pavlov. Basicity of an exponential system and Muckenhaupt's condition. Sov. Math. Dokl. 20 (1979), 655-659. MR 84j:42042

[16] L. Reichel, G. S. Ammar, W. B. Gragg. Discrete least squares approximation by trigonometric polynomials. Math. Comp. 57 (1991), 273-289. MR 91j:65027

[17] K. Seip. On the connection between exponential bases and certain related sequences in $L^{2}(-\pi, \pi)$. J. Functional Anal. 130 (1995), 131-160. MR 96d:46030

[18] T. Strohmer. Efficient methods for digital signal and image reconstruction from non-uniform samples. Ph. D. Thesis, University of Vienna, 1993.

[19] R. Young. An Introduction to Nonharmonic Fourier Series. Academic Press, New York. 1980. MR 81m:42027

[20] A. I. Zayed. Advances in Shannon's sampling theory. CRC Press, 1993. MR 95f:94008

Department of Mathematics, The University of Connecticut, Storrs, CT. 06269-3009

E-mail address: groch@math.uconn.edu 